\title{
Porcelain Gallbladder Decoding the malignant truth
}

Norman O. Machado

$$
\text { الكشف عن الحقيقة الخبيثة الخزفية }
$$

نورمان اونيل ماشادو

ABSTRACT: Gallbladder calcification, also referred to as porcelain gallbladder, has received significant attention in the medical literature due to its perceived role in increasing the risk of developing a gallbladder carcinoma. However, recent reports raise questions challenging this purported high risk. While previous studies reported a concomitant incidence of gallbladder cancer in porcelain gallbladder ranging from $7-60 \%$, more recent analyses indicate the incidence to be much lower (6\%). Based on evidence in the current literature, a prophylactic cholecystectomy is not routinely recommended for all patients with porcelain gallbladder and should be restricted to those with conventional indications, such as young patients. However, it is important to note that a nonoperative approach may require prolonged follow-up. A laparoscopic cholecystectomy is a feasible therapeutic option for patients with porcelain gallbladder, although some researchers have indicated a higher incidence of complications and conversion due to technical difficulties.

Keywords: Gallbladder Diseases; Carcinomas; Cholecystectomy; Physiological Calcification.

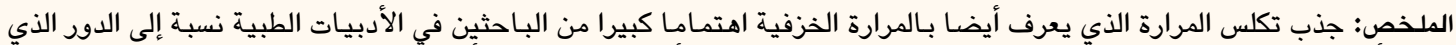

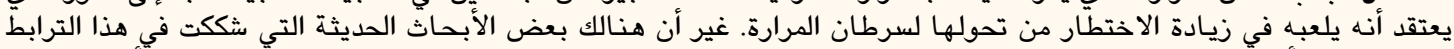

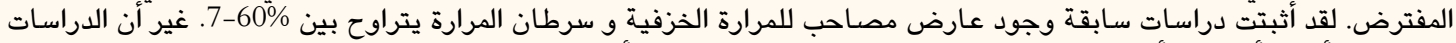

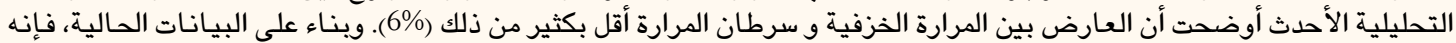

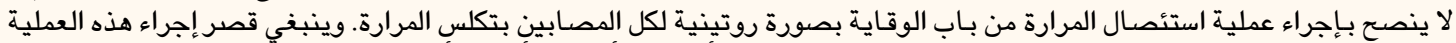

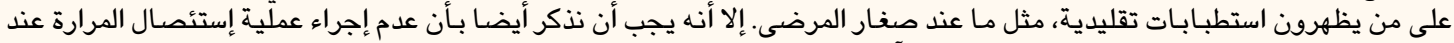

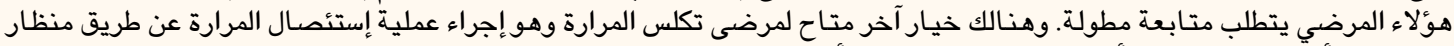

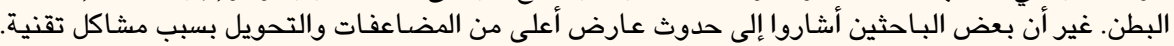
الكلمات المفتاحية: أمراض المرارة؛ سرطانات؛ استئصال المرارة؛ التكلس الفيزيلوجي.

T He term porcelain gallbladder (PGB) is often used to describe calcification of the gallbladder wall. ${ }^{1-3}$ When infiltrated by extensive calcium deposits, the gallbladder wall can become fragile, brittle and bluish in appearance, resulting in a 'porcelain' appearance. ${ }^{2-6}$ There are two distinct types of gallbladder calcification: selective mucosal calcification and diffuse intramural calcification. The latter type is often associated with the traditional description of PGB. ${ }^{1,2}$ The reported overall five-year survival and one-year mortality rates of PGB are $5 \%$ and $88 \%$, respectively. ${ }^{1}$

Historically, PGB was reported to be associated with carcinoma of the gallbladder (CaGB), with the incidence sometimes exceeding $60 \%{ }^{6}$ Since then, a cholecystectomy has become the norm for treating current and preventing future malignancy in patients with PGB. ${ }^{1,2}$ Modern techniques for investigating gallbladder pathology have led to the earlier detection of PGB in comparison to plain X-rays. ${ }^{7,8}$ This change has led to a distortion of the evidence base and a considerable decrease in the rate of detection of CaGB among patients with PGB. ${ }^{1,2,9,10}$ Several recent reports have indicated a much lower incidence of $\mathrm{CaGB}$, thus raising questions regarding the age-old practice of performing a routine cholecystectomy on patients with PGB. ${ }^{2,9,10}$ Moreover, complications following a cholecystectomy are reportedly higher in patients with PGB. ${ }^{10}$ Based on the present evidence in the literature, this review discusses the incidence of PGB, its association with CaGB and the need for a cholecystectomy in patients with PGB.

\section{Risk Factors}

Among 44 patients with PGB, Stephen et al. noted that nonspecific symptoms of a calcified gallbladder included abdominal pain alone (47\%), abdominal pain, 
nausea and vomiting (16\%), abdominal pain and fever (9\%), abdominal pain and jaundice (5\%) and anorexia, nausea and vomiting (5\%); however, $18 \%$ of patients were asymptomatic. ${ }^{1}$ The development of $\mathrm{CaGB}$ in addition to PGB is associated with other risk factors, including gallstones of $>3.0 \mathrm{~cm}$ in size, cholecystoenteric fistulae, an anomalous pancreaticobiliary junction, gallbladder adenomas or polyps, choledochal cysts, occupational exposure to carcinogens and chronic Salmonella typhi infections. ${ }^{1,11}$ Gallbladder cancer is notoriously aggressive, often diagnosed late and has a poor prognosis; hence, there is a need for aggressive treatment for individuals with $\mathrm{PGB}$ and a perceived risk of $\mathrm{CaGB} \cdot{ }^{1,12,13} \mathrm{~A}$ late diagnosis of $\mathrm{CaGB}$ is often due to its nonspecific and variable presentation in the initial stages and the close anatomical relationship of the gallbladder with the liver, which facilitates its spread. ${ }^{1,2}$

\section{Historical Perspective}

The association between gallbladder cancer and calcium deposition on the gallbladder wall was first proposed in $1797 .{ }^{5}$ Since then, several reports have emerged. ${ }^{1-6,9-12,14-18}$ In one study of 4,271 cholecystectomy specimens collected between 1922-1956, adenocarcinomas were noted in two out of 16 calcified gallbladders (12.5\%). ${ }^{5}$ In another report from Argentina, CaGB was detected in 16 out of 26 calcified gallbladder specimens (61.5\%). ${ }^{6}$ In their analysis of 25,900 gallbladder specimens collected between 1962-1999, Stephen et al. found the incidence of gallbladder cancer and calcium deposition to be 7\%. ${ }^{1}$ Interestingly, the incidence and association was related to the type of calcification; gallbladder cancer was restricted to those with selective mucosal calcification (odds ratio [OR]: 13.89; $P=0.01$ ) and no cancer was found in gallbladders with diffuse intramural calcification. ${ }^{1}$ However, a recent literature review suggests that the overall incidence of PGB and gallbladder cancer is $0.2 \%$ and $0.8 \%$, respectively - with a $15 \%$ concomitant incidence-while another report notes the incidence of gallbladder malignancy in patients with gallbladder wall calcification to be $6 \%{ }^{2,9}$

\section{Calcification and Risk of Malignancy}

\section{CALCIFICATION PATTERNS}

The extent of calcification in gallbladders can range from small focal plaques restricted to the mucosal layer and its glandular spaces to the involvement of the full thickness of the gallbladder wall, replacing the muscularis layer with calcified fibrosis and leading to the subsequent denuding of the mucosa. ${ }^{1,2}$ The absence of mucosa in these cases reduces the risk of malignancy. ${ }^{1,4}$ The radiographical appearance of a calcified gallbladder varies based on the extent, degree and location of the calcification. Less intense calcification of the mucosa is unlikely to be identified on a plain X-ray, while more diffuse intramural calcification will appear as a curvilinear or rounded opacity in the right upper quadrant [Figure 1A]. ${ }^{1}$ Recently, the increased use of ultrasonography (US) as part of the investigation process for patients with abdominal discomfort has resulted in the earlier and improved detection of PGB. ${ }^{14}$ Unfortunately, due to its rarity, the natural history and progression of a calcified gallbladder is unknown and could vary based on different patterns of calcification. ${ }^{1,2}$

A definitive diagnosis of PGB can usually be established with an abdominal US or plain noncontrast computed tomography (CT) scan demonstrating the characteristic calcification of the gallbladder wall [Figure 1B]. ${ }^{19}$ In general, US findings are reported as complete or incomplete and have been classified into three types based on the extent and nature of calcification: type I is characterised by a hyperechoic semilunar structure with posterior acoustic shadowing; type II displays a curvilinear echogenic structure with acoustic shadowing; and type III is characterised by irregular clumps of echoes with posterior acoustic shadowing. ${ }^{7,8,13,20}$ While type I corresponds to complete intramural calcification of the gallbladder, types II and III reflect the variations of selective mucosal calcification. ${ }^{8,20}$ In some cases, flecks of mucosal calcification may not be detected during radiological examinations but are found only on pathology. ${ }^{1}$ Khan et al. noted complete transmural and mucosal calcification in $69 \%$ and $23 \%$ of 13 patients with PGB, respectively. ${ }^{9}$

\section{RISK OF MALIGNANCY}

Although the aetiology of gallbladder wall calcification is poorly understood, it is believed to be a consequence of a chronic inflammatory process. ${ }^{4,9}$ Dystrophic calcification and errors in calcium metabolism have been implicated in the formation of PGB and inflammation and ischaemia may progress to transmural calcification. ${ }^{9}$ There is a well-documented association between PGB and the development of CaGB. ${ }^{1,3-6,9,12,14,15}$ Chemicals within stagnant bile or degeneration and regeneration processes within the gallbladder epithelium, leading to mucosal dysplasia, may act as a carcinogenic stimulus. ${ }^{9}$ Most carcinomas associated with $\mathrm{PGB}$ are diffusely infiltrating adenocarcinomas. $^{21}$ 

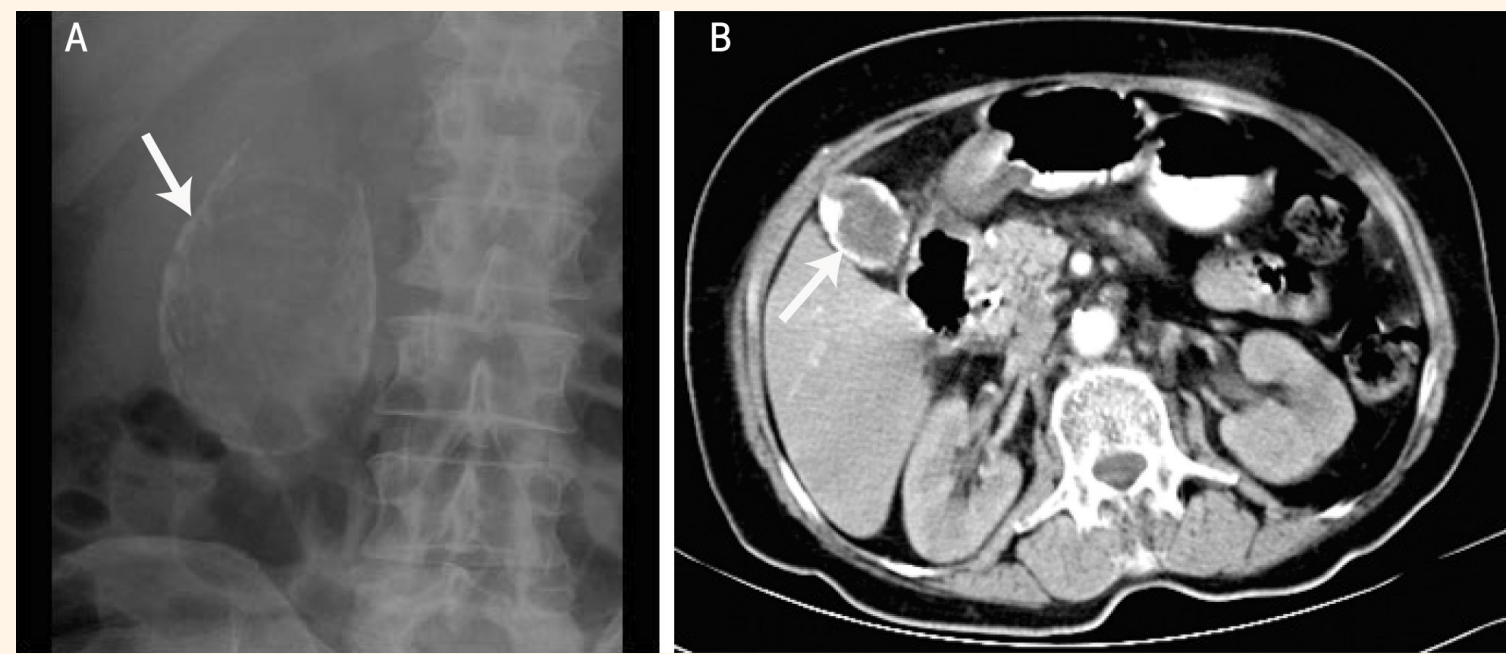

Figure 1: A: Plain X-ray revealing a diffuse intramural calcification (arrow) in a patient with a porcelain gallbladder. B: Computed tomography of the abdomen showing rim calcification of the gallbladder wall (arrow) in a patient with a porcelain gallbladder.

Reproduced with permission from Sen KK, Upadhyaya A, Pimpalwar Y, D'souza J. Cancer in porcelain gallbladder: Rare imaging trait. ${ }^{19}$

A recent systematic review by Schnelldorfer studied the association of PGB with gallbladder cancer; of 111 articles detailing 340 patients with gallbladder wall calcification, the incidence of gallbladder malignancy was $21 \%{ }^{2}$ While the author noted that patients with calcified gallbladders were indeed statistically at risk of developing gallbladder malignancy, on careful analysis of the data, the actual risk of malignancy was significantly less. Moreover, Schnelldorfer indicated certain limitations which could have resulted in an overestimation in the reported incidence of gallbladder cancer among patients with PGB, including: (1) publication bias, in that publishers might prefer to publish rare or 'curious' cases only; (2) selection bias, whereby researchers selected subjects from a population of gallbladder cancer patients rather than from the general population or because most reported patients were published in case series and thus did not include a systematic selection of patients; and (3) sampling bias, as most studies represented institutional experiences of patients with gallbladderrelated symptoms seeking medical advice. ${ }^{2}$ These factors are further compounded by the fact that most gallbladder wall calcifications are asymptomatic, and hence undiagnosed, resulting in an underreporting of benign cases. ${ }^{1,2}$ In a subgroup analysis of 13 studies without obvious selection bias, the rate of gallbladder malignancy was noted to be only $6 \%(0-33 \%)$ in patients with gallbladder calcification compared to $1 \%(0-4 \%)$ in a matched cohort of patients without gallbladder calcification $(P=0.036$; relative risk: 8.0 ; 95\% confidence interval [CI]: $1.0-63.0){ }^{2}$

There has been a recent decline in the reported incidence of $\mathrm{CaGB}$ among patients with PGB
[Table 1]. 1,3,5,6,9,10,16-18,22 A number of potential reasons for this decreasing incidence have been proposed, ranging from possible changes in the natural history of the disease as a consequence of diet or environment to the early detection of calcification due to modern methods for investigating abdominal pain such as US and CT scans. ${ }^{9}$ These factors, along with the increased incidence of laparoscopic cholecystectomies due to the willingness of patients to undergo this less invasive

Table 1: Literature review of case series showing the incidence of porcelain gallbladder with concomitant gallbladder carcinoma $\mathrm{a}^{1,3,5,6,9,10,16-18,22}$

\begin{tabular}{|c|c|c|c|}
\hline \multirow{2}{*}{$\begin{array}{l}\text { Author and year } \\
\text { of case series }\end{array}$} & \multirow[t]{2}{*}{ Samples } & \multicolumn{2}{|c|}{ n (\%) } \\
\hline & & $\begin{array}{l}\text { PGB } \\
\text { cases }\end{array}$ & $\begin{array}{c}\text { Concomitant } \\
\text { PGB and } \\
\text { CaGB cases }\end{array}$ \\
\hline Kapoor et al. ${ }^{16} 2016$ & 116 & 1 & $0(0.0)$ \\
\hline Chen et al..$^{10} 2015$ & - & 192 & $0(0.0)^{*}$ \\
\hline Khan et al. ${ }^{9} 2011$ & 1,200 & $13(1.1)$ & $0(0.0)$ \\
\hline Kim et al. 222009 & 3,159 & $9(0.3)$ & $0(0.0)$ \\
\hline Puia et al. ${ }^{18} 2005$ & 12,000 & $4(0.0)$ & $0(0.0)$ \\
\hline Kwon et al. ${ }^{17} 2004$ & 1,608 & $13(0.8)$ & $1(7.7)$ \\
\hline Stephen et al. ${ }^{1} 2001$ & 25,900 & $44(0.2)$ & $2(4.5)$ \\
\hline Towfigh et al. 2001 & 10,741 & $15(0.1)$ & $0(0.0)$ \\
\hline Etala $^{6} 1962$ & 1,786 & $26(1.5)$ & $16(61.5)$ \\
\hline Cornell et al. 1959 & 4,271 & $16(0.4)$ & $2(12.5)$ \\
\hline Total & 60,781 & $333(0.5)$ & $21(6.3)$ \\
\hline
\end{tabular}

$P G B=$ porcelain gallbladder $C a G B=$ carcinoma in the gallbladder .

*Among the 192 cases of PGB, 102 underwent a cholecystectomy while 90 were observed only; no malignancy was found in either group. 
surgery, may result in the removal of PGBs well before their progression to malignancy. ${ }^{23}$ Moreover, geographical variation in the incidence of $\mathrm{CaGB}$ has been suggested, as the number of cases of concomitant $\mathrm{CaGB}$ and PCB appears to be lower in reports from the USA in comparison to the rest of the world. , $, 6,7,10,17^{2}$

The incidence of gallbladder cancer in incomplete gallbladder calcification has been reported to range from $7-42 \%{ }^{1,24}$ However, additional investigations may be required to detect malignancy if a CT scan does not provide conclusive results. Diffusionweighted magnetic resonance imaging (MRI) has been reported to show hypercellular tumour regions, distinguishing benign and malignant gallbladder lesions; in a recent study, Solak et al. utilised diffusionweighted MRI to reveal CaGB arising from a PGB, identified by linear hyperintense lines due to the hypercellularity of the tumour. ${ }^{7}$ Diffuse malignancies may not be distinguishable from cholecystitis on US and CT scans; moreover, malignant lesions and inflammation can both capture intravenous contrast on a routine MRI scan. ${ }^{7}$ However, diffusion-weighted MRI imaging with fat suppression can delineate the hypercellular tumour regions from cholecystitis. ${ }^{7}$

\section{Potential Predictors of Malignancy}

Most reports suggest that the association between CaGB and PGB can be found in patients with focal mucosal calcification rather than those with extensive calcification and mucosal sloughing. ${ }^{1,8,20}$ However a recent systematic review did not support the view that the depth of calcification is a predictor of malignancy; hence, this factor should not be used as an indicator of the risk of developing malignancy in the future. ${ }^{2}$ Patient age, calcifications limited to a focal area of the gallbladder wall and the absence of stones or microcrystals within the gall bladder lumen were also not found to be a significant risk factors for gallbladder malignancy in PGB. ${ }^{2}$ In terms of clinical presentation, typical symptoms of gallbladder cancer (including painless jaundice, Courvoisier's sign and unexplained weight loss) and a gallbladder mass were the only statistical significant independent predictors of the presence of malignancy (OR: 83.6, 95\% CI: 2.3-2,979.1; $P=0.015$ and OR: 3226.6, 95\% CI: 17.2-603,884.8; $P=0.003$, respectively). ${ }^{2}$ Unfortunately, both typical gallbladder cancer symptoms and gallbladder masses are indicative of advanced malignancy; hence, these indicators are of little use in detecting early malignancy when a curative resection is still feasible. ${ }^{2}$

\section{The Clinician's Role}

Clinicians are often concerned of the risk of malignancy among PGB patients. When these patients are symptomatic, even in the absence of malignancy, a cholecystectomy is a prudent approach. ${ }^{1,2,9}$ However, a dilemma arises when dealing with patients who are asymptomatic, have been diagnosed incidentally and have no features to suggest malignancy. Unfortunately, current evidence from the literature does not indicate predictors for the development of malignancy in the future. ${ }^{1,2,9}$

With an overall risk of malignancy of $0.8-6 \%$ among patients with $\mathrm{PGB}$, the role of a prophylactic cholecystectomy is debatable. ${ }^{2,12,22,25}$ Moreover, the risk of major complications following a laparoscopic cholecystectomy favours a nonoperative approach. ${ }^{1,3,9,22}$ Proponents of a prophylactic cholecystectomy emphasise the benefit of removing the gallbladder and thus eliminating any early undetected malignancies at a potentially curative stage. ${ }^{23,26}$ Such an approach could achieve both a better long-term outcome and avoid the potential legal issues associated with delayed management. Therefore, for patients who are relatively young and fit, a prophylactic cholecystectomy is a reasonable option; however, for those whose perceived risk of perioperative morbidity or mortality is greater than the $6 \%$ risk of developing malignancy, a nonoperative approach is advisable. ${ }^{2}$ Nevertheless, it is important to note that patients who are managed conservatively may require close follow-up with frequent radiological imaging to detect malignancy. In addition, the failure to eject bile due to a calcified gallbladder might lead to gallstone formation. ${ }^{2,9}$ The role of a hepatobiliary scan in assessing the ejection fraction of the gallbladder in such patients is not clear. ${ }^{8}$

During a prophylactic cholecystectomy on patients with PGB, the cholecystectomy specimen should be subjected to frozen section/histopathological examination. If this reveals a malignancy, then a conversion to an extended/radical cholecystectomy should be carried out by wedge resection of liver tissue and the gallbladder bed, followed by a lymphadenectomy. ${ }^{16}$ In a recent report, Kapoor et al. recommended an anticipatory extended cholecystectomy in patients whose gallbladder wall thickness was $>3 \mathrm{~mm}$ on radiological imaging and in whom the possibility of malignancy could not be ruled out. ${ }^{16}$ This procedure involves the removal of a $2 \mathrm{~cm}$ wedge of segments $\mathrm{IVb}$ and $\mathrm{V}$ of the adjoining liver and a subsequent lymphadenectomy if the frozen section confirms malignancy. Using this approach, the cholecystohepatic plane is not breached and it is possible to perform an 
en masse removal of the malignant lesion; resection of the adjoining liver en masse prevents the possible microscopic infiltration of gallbladder cancer into the liver. ${ }^{16}$

\section{LAPAROSCOPIC CHOLECYSTECTOMIES}

Patients with PGB are generally not considered ideal patients for a laparoscopic cholecystectomy because of the brittle nature of the calcified gallbladder and the difficulty in grasping and dissecting it. ${ }^{1}$ However, in the absence of evidence of malignancy, successful laparoscopic cholecystectomies (including single-port procedures) have been reported, with a conversion rate to open surgery of $5-25 \% .{ }^{9,10,21}$ In their case series, Khan et al. indicated that the main cause of conversion was an inability to obtain an adequate critical view of the cystic duct and artery. ${ }^{9}$

In most patients, the risk of cholecystectomyrelated complications is relatively low, with major complications occurring in 3-4\% of cases, including perioperative mortality and common bile duct injuries (0.5\% each). ${ }^{2,27}$ However, the risk may be significantly higher in patients with adverse gallbladder conditions, such as recurrent inflammation or cirrhosis, or in individuals with significant comorbidities, particularly those of a cardiac or respiratory nature. ${ }^{27,28}$ In a recent report, perioperative complications following a cholecystectomy in patients with PGB varied based on the presence of symptoms prior to the surgery; the rate of complications was $10.7 \%$ among those who were asymptomatic and $16.7 \%$ among those who were symptomatic. ${ }^{15}$ Moreover, complications led to eight endoscopic or percutaneous interventions and five additional surgeries. ${ }^{15}$

\section{Conclusion}

In summary, recent evidence has shown that that the potential risk of developing malignancies among patients with PGB is much lower than previously believed. However, there are as yet no clinical indicators to suggest which patients might develop malignancies in the future. Typical symptoms which are suggestive of gallbladder cancer are of little clinical benefit as they tend to indicate advanced malignancy with a poor prognosis. The optimal management for symptomatic patients with $\mathrm{PGB}$ or individuals who are young and fit is a prophylactic cholecystectomy. For those with a perioperative risk of complications or mortality greater than the risk of developing malignancy, a prophylactic cholecystectomy is not recommended. However, a nonoperative approach may subsequently require close follow-up.

\section{References}

1. Stephen AE, Berger DL. Carcinoma in the porcelain gallbladder: A relationship revisited. Surgery 2001; 129:699-703. doi: 10.10 67/msy.2001.113888.

2. Schnelldorfer T. Porcelain gallbladder: A benign process or concern for malignancy? J Gastrointest Surg 2013; 17:1161-8. doi: 10.1007/s11605-013-2170-0.

3. Towfigh S, McFadden DW, Cortina GR, Thompson JE Jr, Tompkins RK, Chandler C, et al. Porcelain gallbladder is not associated with gallbladder carcinoma. Am Surg 2001; 67:7-10.

4. Polk HC Jr. Carcinoma and the calcified gall bladder. Gastroenterology 1966; 50:582-5. doi: 10.1016/S0016-5085 (66)80037-9

5. Cornell CM, Clarke R. Vicarious calcification involving the gallbladder. Ann Surg 1959; 149:267-72. doi: 10.1097/00000658195902000-00013.

6. Corsetti RL, Wanebo HJ. Gallbladder cancer. In: Cameron JL, Ed. Current Surgical Therapy, 5th edition. St. Louis, Missouri, USA: Mosby, 1998. Pp. 462-8

7. Solak A, Solak I, Genç B, Sahin N. The role of diffusion-weighted examination in non-polyploid gallbladder malignancies: A preliminary study. Turk J Gastroenterol 2013; 24:148-53. doi: 10.4318/tjg.2013.0659.

8. Kane RA, Jacobs R, Katz J, Costello P. Porcelain gallbladder: Ultrasound and CT appearance. Radiology 1984; 152:137-41. doi: 10.1148/radiology.152.1.6729103.

9. Khan ZS, Livingston $\mathrm{EH}$, Huerta S. Reassessing the need for prophylactic surgery in patients with porcelain gallbladder: Case series and systematic review of the literature. Arch Surg 2011; 146:1143-7. doi: 10.1001/archsurg.2011.257.

10. Chen GL, Akmal Y, DiFronzo AL, Vuong B, O'Connor V. Porcelain gallbladder: No longer an indication for prophylactic cholecystectomy. Am Surg 2015; 81:936-40.

11. Nagorney DM, McPherson GA. Carcinoma of the gallbladder and extrahepatic bile ducts. Semin Oncol 1988; 15:106-15.

12. Rana SS, Bhasin DK. Porcelain gall bladder on endoscopic ultrasound. Endosc Ultrasound 2013; 2:112-13. doi: 10.4103/ 2303-9027.117702

13. Rakić M, Patrlj L, Kopljar M, Kliček R, Kolovrat M, Loncar B, et al. Gallbladder cancer. Hepatobiliary Surg Nutr 2014; 3:221-6. doi: 10.3978/j.issn.2304-3881.2014.09.03

14. Gómez-López JR, De Andrés-Asenjo B, Ortega-Loubon C. A porcelain gall bladder and a rapid tumor dissemination. Ann Med Surg (Lond) 2014; 3:119-22. doi: 10.1016/j.amsu. 2014.09.002.

15. Ansari S. Porcelain gallbladder. Indian J Gastroenterol 2014; 33:399. doi: 10.1007/s12664-013-0362-x.

16. Kapoor VK, Singh R, Behari A, Sharma S, Kumar A, Prakash A, et al. Anticipatory extended cholecystectomy: The 'Lucknow' approach for thick walled gall bladder with low suspicion of cancer. Chin Clin Oncol 2016; 5:8. doi: 10.3978/j.issn.23043865.2016.02.07

17. Kwon AH, Inui H, Matsui Y, Uchida Y, Hukui J, Kamiyama Y. Laparoscopic cholecystectomy in patients with porcelain gallbladder based on the preoperative ultrasound findings. Hepatogastroenterology 2004; 51:950-3.

18. Puia IC, Vlad L, Iancu C, Al-Hajjar N, Pop F, Bălă O, et al. [Laparoscopic cholecystectomy for porcelain gallbladder]. Chirurgia (Bucur) 2005; 100:187-9.

19. Sen KK, Upadhyaya A, Pimpalwar Y, D'souza J. Cancer in porcelain gallbladder: Rare imaging trait. Indian J Cancer 2011; 48:132-4. doi: 10.4103/0019-509X.76646. 
20. Shimizu M, Miura J, Tanaka T, Itoh H, Saitoh Y. Porcelain gallbladder: Relation between its type by ultrasound and incidence of cancer. J Clin Gastroenterol 1989; 11:471-6. doi: 10.1097/00004836-198908000-00027.

21. Shivanna Puttasubbappa P, Pallavi P. Porcelain gall bladder mimicking carcinoma gallbladder: A case report and review of literature. Indian J Surg 2013; 75:208-9. doi: 10.1007/s12262012-0545-1.

22. Kim JH, Kim WH, Yoo BM, Kim JH, Kim MW. Should we perform surgical management in all patients with suspected porcelain gallbladder? Hepatogastroenterology 2009; 56:943-5.

23. Cavallaro A, Piccolo G, Panebianco V, Lo Menzo E, Berretta M, Zanghì $\mathrm{A}$, et al. Incidental gallbladder cancer during laparoscopic cholecystectomy: Managing an unexpected finding. World J Gastroenterol 2012; 18:4019-27. doi: 10.3748/ wjg.v18.i30.4019.

24. Sheth S, Bedford A, Chopra S. Primary gallbladder cancer: Recognition of risk factors and the role of prophylactic cholecystectomy. Am J Gastroenterol 2000; 95:1402-10. doi: 10.1111/j.1572-0241.2000.02070.x.
25. Igami T, Usui H, Ebata T, Yokoyama Y, Sugawara G, Takahashi Y, et al. Single-incision laparoscopic cholecystectomy for porcelain gallbladder: A case report. Asian J Endosc Surg 2013; 6:52-4. doi: 10.1111/ases.12008.

26. Gollan JL, Bulkley GB, Diehl AM. National Institutes of Health Consensus Conference: Gallstones and laparoscopic cholecystectomy. JAMA 1993; 269:1018-24. doi: 10.1001/ jama.1993.03500080066034.

27. Machado NO. Biliary complications postlaparoscopic cholecystectomy: Mechanism, preventive measures, and approach to management: A review. Diagn Ther Endosc 2011; 2011:967017. doi: 10.1155/2011/967017.

28. Machado NO. Laparoscopic cholecystectomy in cirrhotics. JSLS 2012; 16:392-400. doi: 10.4293/108680812X13462882736493. 